Supporting Information

\title{
Proton-Coupled Electron Transfer Reduction of a Quinone by an Oxide-Bound Riboflavin Derivative
}

\author{
Na Song, Christopher J. Dares, Matthew V. Sheridan and Thomas J. Meyer*
}

University of North Carolina at Chapel Hill, Chapel Hill, NC 27599-3290

tjmeyer@unc.edu 

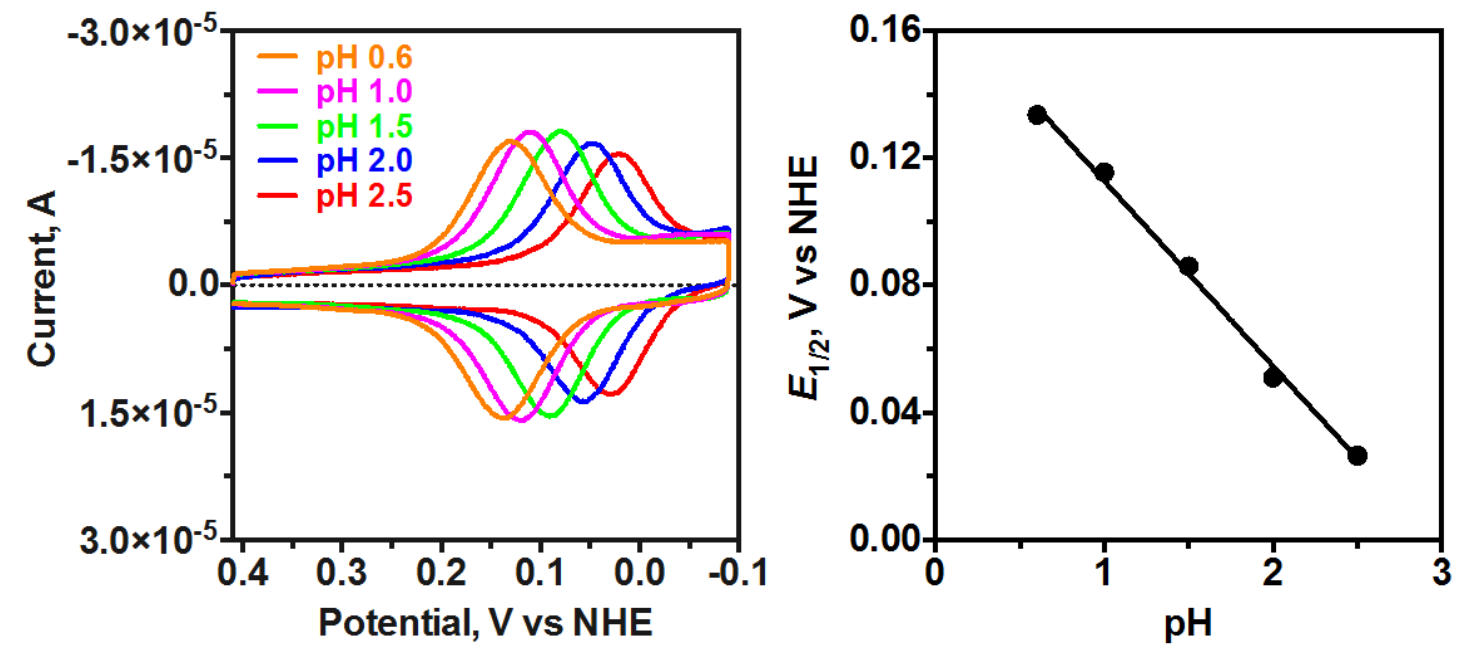

Figure S1. FTO|-FMN in 1:1 $\mathrm{CH}_{3} \mathrm{CN} / \mathrm{H}_{2} \mathrm{O}(\mathrm{V}: \mathrm{V})$, $\mathrm{pH} 0.6-2.5\left(\mathrm{HClO}_{4}\right), I=0.25 \mathrm{M}\left(\mathrm{NaClO}_{4}\right)$, scan rate 0.1 $\mathrm{V} / \mathrm{s}$.

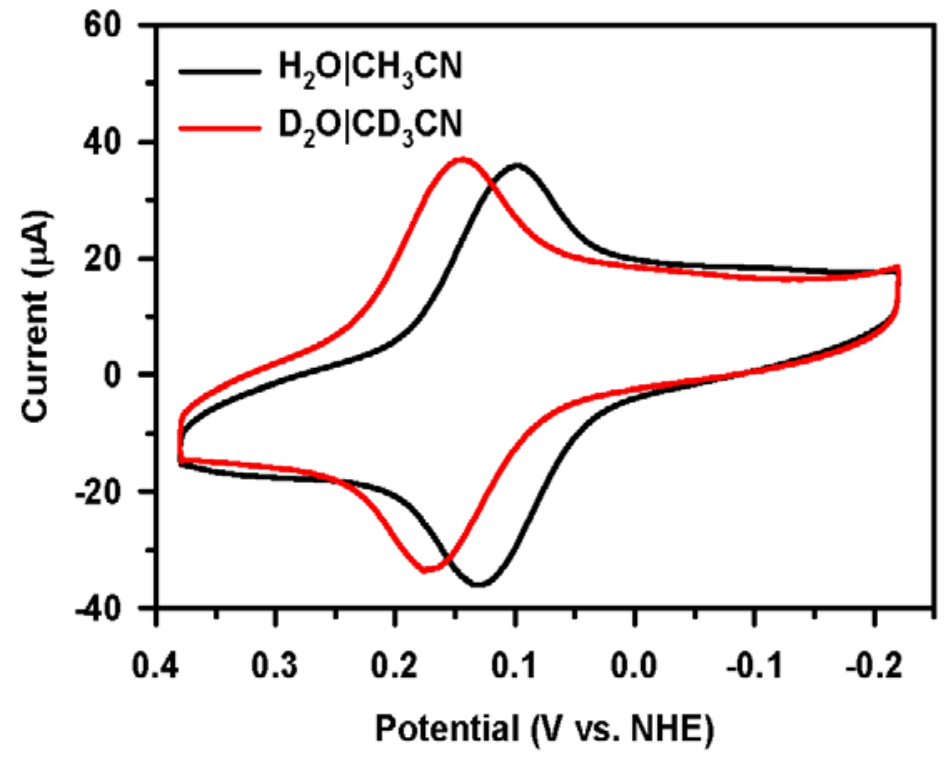

Figure S2. FTO|-FMN in 1:1 $\mathrm{CH}_{3} \mathrm{CN} / \mathrm{H}_{2} \mathrm{O}(\mathrm{V}: \mathrm{V})$ (Black), and in 1:1 $\mathrm{CD}_{3} \mathrm{CN} / \mathrm{D}_{2} \mathrm{O}(\mathrm{V}: \mathrm{V})(\mathrm{Red}),\left[\mathrm{HClO}_{4}\right]=$

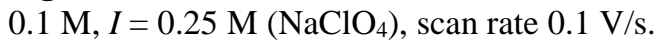



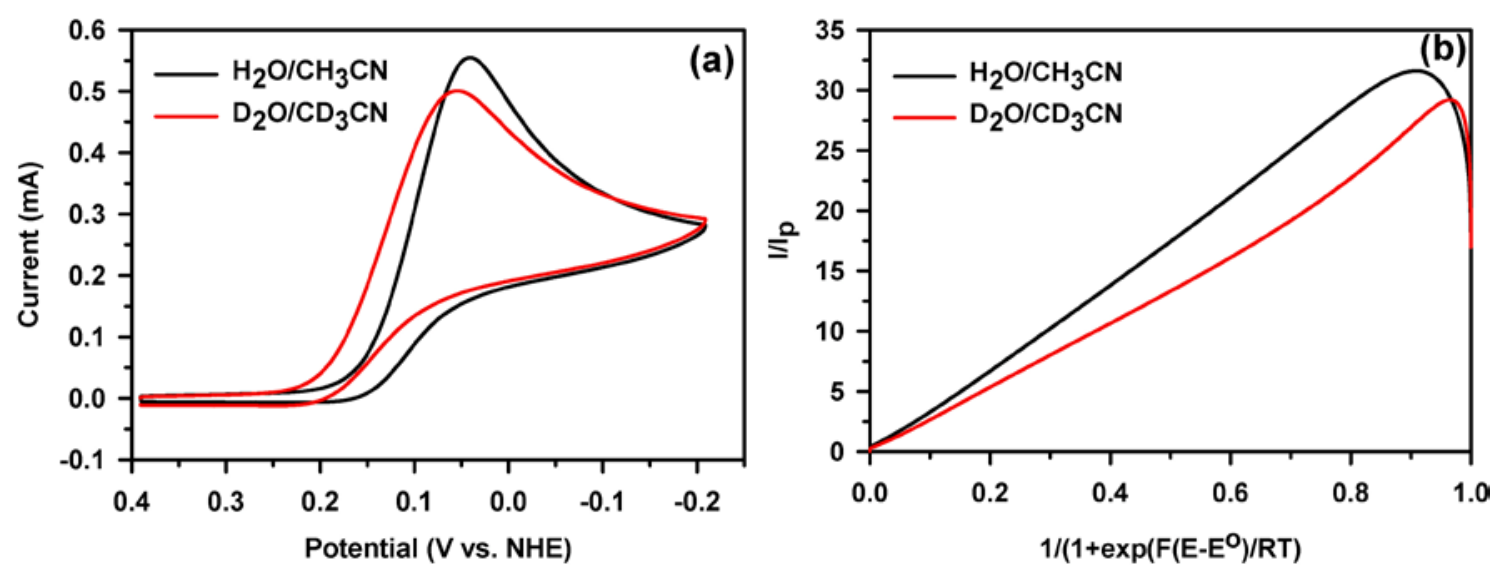

Figure S3. FTO|-FMN in 1:1 $\mathrm{CH}_{3} \mathrm{CN} / \mathrm{H}_{2} \mathrm{O}(\mathrm{V}: \mathrm{V})$ (Black), and 1:1 $\mathrm{CD}_{3} \mathrm{CN} / \mathrm{D}_{2} \mathrm{O}(\mathrm{V}: \mathrm{V})$ (Red), $\left[\mathrm{HClO}_{4}\right]=0.1$ $\mathrm{M}, I=0.25 \mathrm{M}\left(\mathrm{NaClO}_{4}\right)$, and $1 \mathrm{mM} \mathrm{Me} 4 \mathrm{Q}$; scan rate $0.1 \mathrm{~V} / \mathrm{s}$. Left: Cyclic voltammograms; Right: Foot-ofthe-wave analysis.

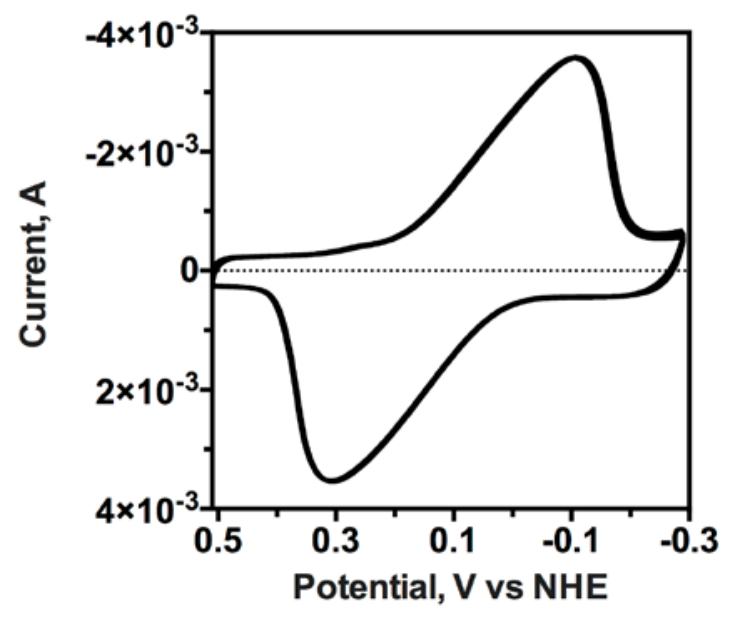

Figure S4. CV of nanoITO|-FMN in 1:1 $\mathrm{CH}_{3} \mathrm{CN} / \mathrm{H}_{2} \mathrm{O}(\mathrm{V}: \mathrm{V})$, $\left[\mathrm{HClO}_{4}\right]=0.1 \mathrm{M}, I=0.25 \mathrm{M}\left(\mathrm{NaClO}_{4}\right)$, scan rate $0.1 \mathrm{~V} / \mathrm{s}, 300$ cycles. 

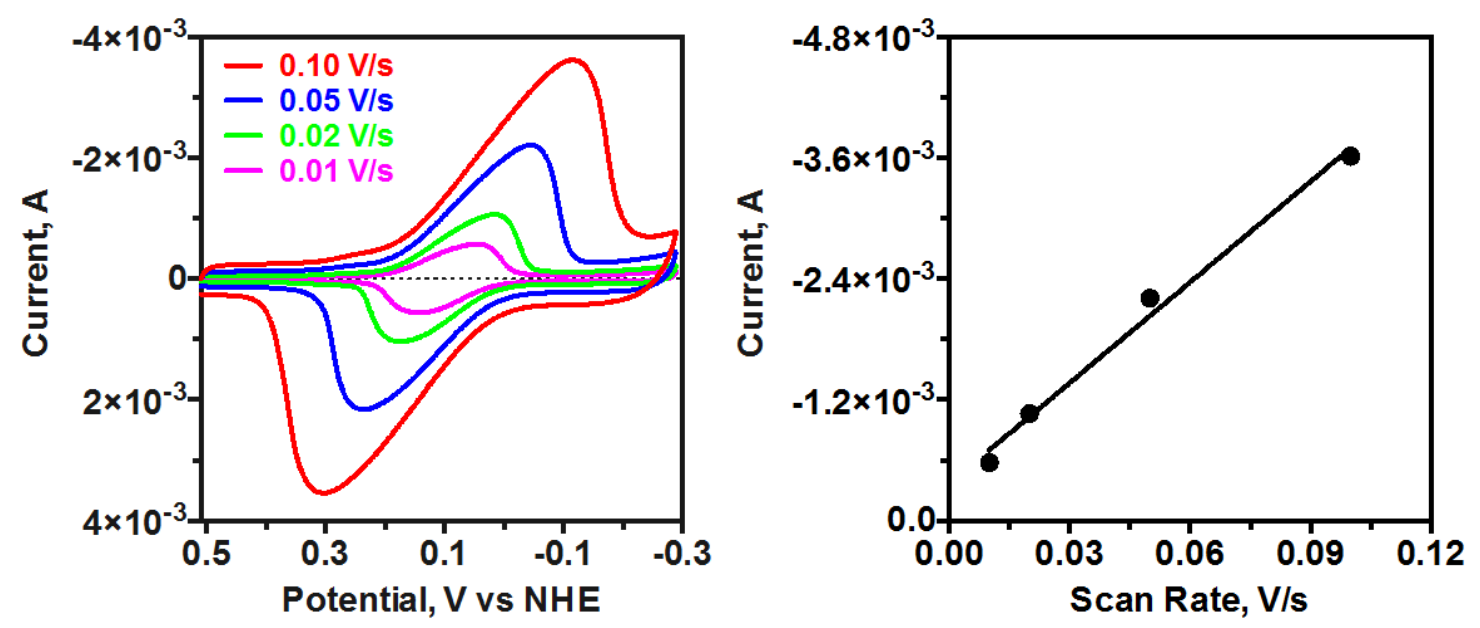

Figure S5. nanoITO|-FMN in 1:1 $\mathrm{CH}_{3} \mathrm{CN} / \mathrm{H}_{2} \mathrm{O}(\mathrm{V}: \mathrm{V})$, $\left[\mathrm{HClO}_{4}\right]=0.1 \mathrm{M}, I=0.25 \mathrm{M}\left(\mathrm{NaClO}_{4}\right)$ at scan rates $0.01,0.02,0.05,0.1 \mathrm{~V} / \mathrm{s}$.

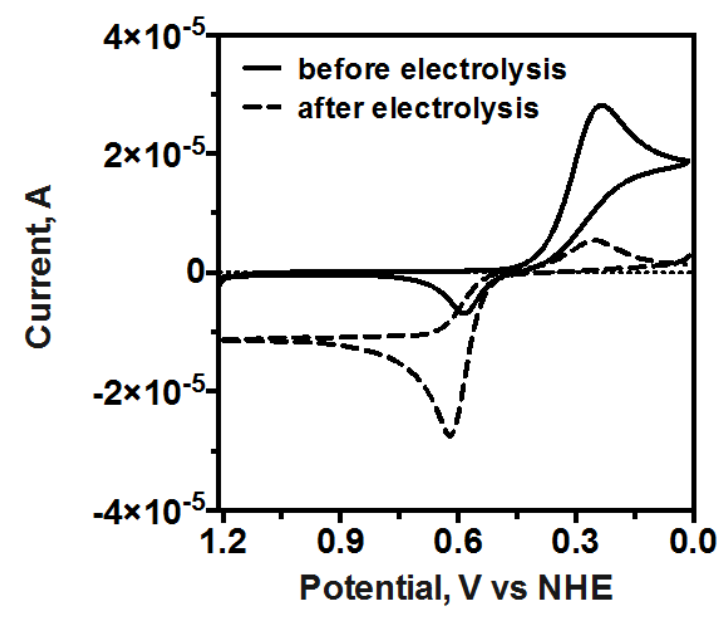

Figure S6. CVs of $2.6 \mathrm{mM} \mathrm{Me}_{4} \mathrm{Q}$ before (solid line) and after (dashed line) electrolysis at glassy

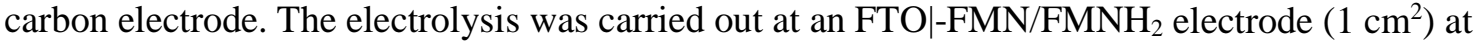
$0.11 \mathrm{~V}$ vs NHE in $1: 1 \mathrm{CH}_{3} \mathrm{CN} / \mathrm{H}_{2} \mathrm{O}(\mathrm{V}: \mathrm{V}),\left[\mathrm{HClO}_{4}\right]=0.1 \mathrm{M}, I=0.25 \mathrm{M}\left(\mathrm{NaClO}_{4}\right)$. 


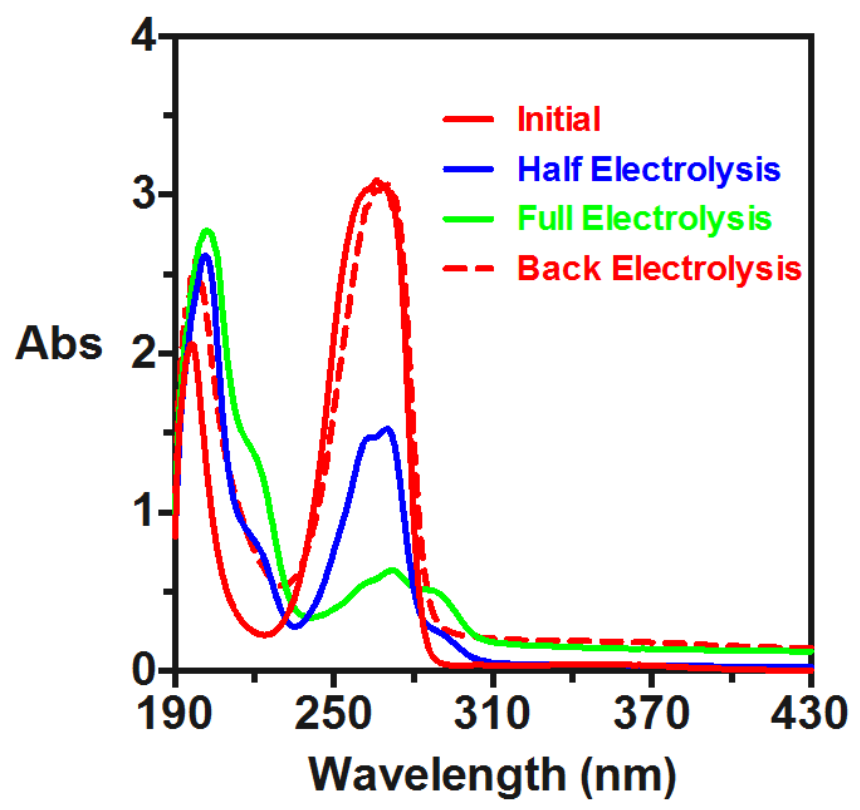

Figure S7. UV-vis of the solution throughout the electrolysis $(0.17 \mathrm{mM}$ analyte) in $1: 1$ $\mathrm{CH}_{3} \mathrm{CN} / \mathrm{H}_{2} \mathrm{O}(\mathrm{V}: \mathrm{V}),\left[\mathrm{HClO}_{4}\right]=0.1 \mathrm{M}, \mathrm{I}=0.25 \mathrm{M}\left(\mathrm{NaClO}_{4}\right)$. 